HD-THEP-94-2

\title{
Flow Equations and BRS Invariance for Yang-Mills Theories
}

\author{
Ulrich Ellwanger円 \\ Institut für Theoretische Physik \\ Universität Heidelberg \\ Philosophenweg 16, D-69120 Heidelberg, FRG
}

\begin{abstract}
:
Flow equations describe the evolution of the effective action $\Gamma_{k}$ in the process of varying an infrared cutoff $k$. The presence of the infrared cutoff explicitly breaks gauge and hence BRS invariance. We derive modified Slavnov-Taylor identities, which are valid for nonvanishing $k$. They guarantee the BRS invariance of $\Gamma_{k}$ for $k \rightarrow 0$, and hence allow the study of non-abelian gauge theories by integrating the flow equations. Within a perturbative expansion of $\Gamma_{k}$, we derive an equation for a $k$ dependent mass term for the gauge fields implied by the modified Slavnov-Taylor identities.
\end{abstract}

\footnotetext{
${ }^{1}$ Supported by a DFG Heisenberg fellowship; e-mail: I96 at VM.URZ.UNI-HEIDELBERG.DE
} 
The use of flow equations (or exact renormalization group equations [1] resp. evolution equations) in continuum quantum field theory has recently been the subject of numerous investigations. They were employed by Polchinski and many others [2]-[6] in order to simplify proofs of perturbative renormalizability. Here the flow equations are used to construct bare actions (depending on an ultraviolet cutoff $\Lambda$ ), which guarantee the existence of finite quantum effective actions in the limit $\Lambda \rightarrow \infty$.

Alternatively the flow equations can be used to construct quantum effective actions in terms of bare actions, with an ultraviolet cutoff $\Lambda$ kept fixed. Their integration with respect to an infrared cutoff $k$ then serves as a computational tool, which allows to calculate the generating functionals (or the Green functions) in the limit $k \rightarrow 0$ in terms of boundary conditions for those functionals at some large infrared cutoff $k=\bar{k}$. The result corresponds to the one of a quantum field theory with fixed ultraviolet cutoff $\Lambda=\bar{k}$, with a classical action related to the boundary conditions at $k=\bar{k}$. For recent work in this direction see refs. [7], [8].

The simple form and the exactness of the flow equations is based on the fact that the cutoffs are introduced by modifying the propagators of the fields. In momentum space, e.g., the propagators get multiplied by a function of $p^{2}$, which vanishes rapidly for momenta beyond the cutoffs. The application of the present concept of flow equations to gauge theories leads thus to serious problems, since the presence of such cutoffs breaks gauge invariance explicitly. (Kleppe and Woodard [9] studied a closely related regularization which affects not just the propagators, but modifies the entire action. This method preserves distorted versions of gauge symmetries, but the resulting effective action no longer satisfies simple flow equations.)

In [8] background gauge fields were introduced in order to cope with this situation, but their presence in the final expressions for the generating functionals leads to new conceptual and practical difficulties. In [4]-[6] Ward or Slavnov-Taylor (ST) identities [10] were employed in order to obtain proofs of perturbative renormalizability for gauge theories.

Let us denote by $G_{k}^{\Lambda}(J), \Gamma_{k}^{\Lambda}(\phi)$ the generating functionals of connected and oneparticle irreducible Green functions, respectively. The indices $k$ and $\Lambda$ refer to the fact that only modes with $k^{2}<p^{2}<\Lambda^{2}$ have been integrated out, i.e. $G_{k}^{\Lambda}$ and $\Gamma_{k}^{\Lambda}$ are computed in the presence of an infrared cutoff $k$ and an ultraviolet cutoff $\Lambda$ in the propagator. For $k=\Lambda G_{\Lambda}^{\Lambda}$ and $\Gamma_{\Lambda}^{\Lambda}$ are simply related to a bare action $S_{0}(\Lambda)$ [3], [7]. Proofs of perturbative renormalizability start with the construction of $G_{\Lambda}^{\Lambda}$ resp. $\Gamma_{\Lambda}^{\Lambda}$ and hence $S_{0}(\Lambda)$, given the knowledge of the relevant or marginal couplings of $G_{0}^{\Lambda}$ and $\Gamma_{0}^{\Lambda}$, by integrating the flow equations for $G_{k}^{\Lambda}$ resp. $\Gamma_{k}^{\Lambda}$. Subsequently the limit $\Lambda \rightarrow \infty$ has to be shown to exist, keeping $G_{0}^{\Lambda}$ and $\Gamma_{0}^{\Lambda}$ finite. In [4]- [6] Ward or Slavnov-Taylor (ST) identities were imposed on the relevant or marginal couplings of $G_{0}^{\Lambda}$ resp. $\Gamma_{0}^{\Lambda}$, and it was argued, that a bare action $S_{0}(\Lambda)$ consistent with these identities can be constructed.

This procedure is of no help, however, if we want to compute the generating functionals for $k \rightarrow 0$ in terms of $S_{0}(\Lambda)$; we do not know the relevant or marginal couplings of $G_{0}^{\Lambda}$ resp. $\Gamma_{0}^{\Lambda}$ beforehand. It should be clear that $G_{\Lambda}^{\Lambda}$ resp. $\Gamma_{\Lambda}^{\Lambda}$ and hence $S_{0}(\Lambda)$ cannot satisfy the standard ST identies, which are related to BRS 
invariance [11] and hence to gauge symmetry. We need a condition on the breaking on BRS symmetry of $G_{\Lambda}^{\Lambda}$ resp. $\Gamma_{\Lambda}^{\Lambda}$ and hence $S_{0}(\Lambda)$, which compensates precisely the breaking of BRS symmetry induced by the integration over modes with a finite cutoff $\Lambda$. Only such a form of $S_{0}(\Lambda)$ can guarantee the validity of the standard ST identities for $G_{0}^{\Lambda}$ resp. $\Gamma_{0}^{\Lambda}$ and hence the unitarity of the theory.

Below we derive such a condition in the form of modified ST identities for $G_{k}^{\Lambda}$ resp. $\Gamma_{k}^{\Lambda}$, which enjoy the following properties:

1. If they are satisfied for some particular value of $k$, e.g. for $k=\bar{k}$, they are automatically satisfied for any other value of $k$, provided $G_{k}^{\Lambda}$ and $\Gamma_{k}^{\Lambda}$ are related to $G_{\bar{k}}^{\Lambda}$ and $\Gamma_{\bar{k}}^{\Lambda}$ by integrating the flow equations.

2. In the limit $k \rightarrow 0$ they turn into the standard ST identities.

Hence, if we require the boundary conditions $G_{\bar{k}}^{\Lambda}$ or $\Gamma_{\bar{k}}^{\Lambda}$ to satisfy the modified ST identities, the BRS invariance of $G_{k=0}^{\Lambda}$ and $\Gamma_{k=0}^{\Lambda}$ is guaranteed.

In fact these modified ST identities are closely related to the "fine tuning condition" for the effective Lagrangian derived and studied by Becchi [5]. There the fine-tuning condition was analyzed with the aim to construct gauge symmetry violating counter terms, which compensate precisely for the breaking of gauge symmetry induced by the integration over modes with a finite cutoff $\Lambda$, and solutions were shown to exist.

Below we will construct a perturbative solution of the modified ST identities. We will be concerned with the necessary presence of a mass term for the gauge fields within $\Gamma_{k}$, for a pure $S U(N)$ Yang Mills theory, for $k \neq 0$ due to the modified ST identities.

A priori we could just present the flow equations together with the form of the modified ST identities, and show that they satisfy both conditions 1 . and 2 . above. Since the perturbative solutions of the flow equations have been shown to correspond to the standard perturbative expansion within various contexts [3] - [8], this constitutes a self-consistent definition of the theory at the perturbative level.

Instead of this ad hoc presentation we prefer to motivate the explicit form of the modified ST identities in a way which, however, implicitely assumes the existence of an invariant UV regularisation. Its existence might be doubted; this is irrelevant, however, since we do not have to rely on it in order to define the theory. The final properties of the flow equations and the modified ST identities are independent of the form of the derivation and the underlying assumptions.

Let us first specify our conventions for a classical Yang-Mills theory. (Throughout this paper we work in Euclidean space with arbitrary dimension d.) The Yang-Mills action is given by

$$
S_{Y M}=\frac{1}{4} \int d^{d} x F_{\mu \nu}^{a} F_{\mu \nu}^{a}
$$

with

$$
F_{\mu \nu}^{a}=\partial_{\mu} A_{\nu}^{a}-\partial_{\nu} A_{\mu}^{a}+g f_{b c}^{a} A_{\mu}^{b} A_{\nu}^{c} .
$$

The gauge-fixing part reads

$$
S_{\mathrm{gf}}=\frac{1}{2 \alpha} \int d^{d} x \partial_{\mu} A_{\mu}^{a} \partial_{\nu} A_{\nu}^{a}
$$


and the ghost action is given by

$$
S_{\mathrm{gh}}=\int d^{d} x \partial_{\mu} \bar{c}^{a}\left(\delta_{a c} \partial_{\mu}+g f_{b c}^{a} A_{\mu}^{b}\right) c^{c} .
$$

In order to describe the BRS transformations it is convenient to introduce operators $O_{A, \mu}^{a}$ and $O_{g}^{a}$, which are related to the BRS variations of $A_{\mu}^{a}$ and $c^{a}$, respectively:

$$
\begin{gathered}
O_{A, \mu}^{a}=\partial_{\mu} c^{a}+g f_{b c}^{a} A_{\mu}^{b} c^{c}, \\
O_{g}^{a}=\frac{1}{2} g f_{b c}^{a} c^{b} c^{c} .
\end{gathered}
$$

Note that the ghosts $c, \bar{c}$ and hence the operator $O_{A}$ are Grassmann-valued. The sum $S_{Y M}+S_{\mathrm{gf}}+S_{\mathrm{gh}}$ is not gauge-invariant, but invariant under BRS transformations of $A_{\mu}^{a}, c^{a}$ and $\bar{c}^{a}$ employing a Grassmann parameter $\zeta$ :

$$
\begin{aligned}
& \delta A_{\mu}^{a}=O_{A, \mu}^{a} \zeta \\
& \delta c^{a}=O_{g}^{a} \zeta \\
& \delta \bar{c}^{a}=-\frac{1}{\alpha} \partial_{\mu} A_{\mu} \zeta .
\end{aligned}
$$

Note that the operators $O_{A}$ and $O_{g}$ are BRS-invariant as well.

The standard definition of the generating functional $G_{k}$ of connected Green functions makes use of sources $J_{\mu}{ }^{a}, \chi^{a}$ and $\bar{\chi}^{a}$ for the fields $A_{\mu}{ }^{a}, \bar{c}^{a}$ and $c^{a}$, respectively, and additional sources $K_{\mu}{ }^{a}$ and $L^{a}$ coupled to the operators $O_{A, \mu}^{a}$ and $O_{g}^{a}$. (The sources $\chi^{a}, \bar{\chi}^{a}$ and $K_{\mu}^{a}$ will be Grassmann-valued.) We also introduce a term $\Delta S_{k}$, which will generate an infrared cutoff both for the gauge fields and the ghosts. The expression for $G_{k}$ then becomes

$$
e^{-G_{k}(J, \chi, \bar{\chi}, K, L)}=\int \mathcal{D}_{R e g}(A, c, \bar{c}) e^{-\left(S_{Y M}+S_{\mathrm{gf}}+S_{\mathrm{gh}}+\Delta S_{k}+S_{c . t .}\right)+J \cdot A+\bar{\chi} \cdot c+\chi \cdot \bar{c}+K \cdot O_{A}+L \cdot O_{g}}
$$

Here we have assumed the existence of an ultraviolet regularization (indicated by the index "Reg" attached to the path integral measure) and counter terms (present in $S_{\text {c.t. }}$ ), which should be a) invariant under BRS transformations, b) independent of the infrared cutoff $k$. As mentioned above, an ultraviolet regularisation with these properties might not even exist; below, however, we will just require a definition of the functionals of the theory in terms of solutions of the flow equations rather than the existence of the path integral (8).

From now on we prefer to work in momentum space; expressions like $J \cdot A$ etc are to be read as

$$
J \cdot A \equiv \int D p J_{\mu}{ }^{a}(-p) A_{\mu}^{a}(p) ; \quad D p \equiv \frac{d^{d} p}{(2 \pi)^{d}} .
$$

The explicit form of the infrared cutoff term $\Delta S_{k}$ is given by

$$
\Delta S_{k}=\int D p\left[\frac{1}{2} A_{\mu}^{a}(-p) R_{\mu \nu}^{k}\left(p^{2}\right) A_{\nu}^{a}(p)+\bar{c}^{a}(-p) \tilde{R}^{k}\left(p^{2}\right) c^{a}(p)\right] .
$$


The functions $R_{\mu \nu}^{k}\left(p^{2}\right)$ (which will depend on the gauge parameter $\alpha$ ) and $\tilde{R}^{k}\left(p^{2}\right)$ modify the gauge field and ghost propagators such that the propagation of modes with $p^{2}<k^{2}$ is suppressed. Convenient choices are

$$
\begin{aligned}
R_{\mu \nu}^{k}\left(p^{2}\right) & =\left(p^{2} \delta_{\mu \nu}+\left(\frac{1}{\alpha}-1\right) p_{\mu} p_{\nu}\right) R^{k}\left(p^{2}\right), \\
\tilde{R}^{k}\left(p^{2}\right) & =p^{2} R^{k}\left(p^{2}\right),
\end{aligned}
$$

where $R^{k}\left(p^{2}\right)$ vanishes for $p^{2} \gg k^{2}$ and diverges for $p^{2} \ll k^{2}$. With (11) the gauge field propagator $P_{A, \mu \nu}$ and the ghost propagator $P_{g}$ become

$$
\begin{aligned}
P_{A, \mu \nu}(p) & =\left[\frac{\delta_{\mu \nu}}{p^{2}}+(\alpha-1) \frac{p_{\mu} p_{\nu}}{p^{4}}\right] \cdot \frac{1}{1+R^{k}\left(p^{2}\right)}, \\
P_{g}(p) & =\frac{1}{p^{2}} \cdot \frac{1}{1+R^{k}\left(p^{2}\right)} .
\end{aligned}
$$

A possible explicit form for $R^{k}\left(p^{2}\right)$ is given by

$$
R^{k}\left(p^{2}\right)=\frac{e^{-\left(p^{2} / k^{2}\right)}}{1-e^{-\left(p^{2} / k^{2}\right)}} .
$$

Now we use the representation eq. (8) for $G_{k}$ in order to motivate the corresponding flow equations. We simply have to differentiate both sides with respect to $k$. On the r.h.s. we obtain an expectation value of $\left(-\partial_{k} \Delta S_{k}\right)$, which is quadratic in the fields according to eq. (10). After replacing the fields by variations with respect to the corresponding sources, which allows to pull the expressions out of the path integral, the flow equation for $G_{k}(J, \chi, \bar{\chi}, K, L)$ becomes

$$
\begin{aligned}
\partial_{k} G_{k}= & \int D p\left\{\frac{1}{2} \partial_{k} R_{\mu \nu}^{k}\left(p^{2}\right)\left[\frac{\delta G_{k}}{\delta J_{\mu}^{a}(p)} \frac{\delta G_{k}}{\delta J_{\nu}^{a}(-p)}-\frac{\delta^{2} G_{k}}{\delta J_{\mu}^{a}(p) \delta J_{\nu}^{a}(-p)}\right]\right. \\
& \left.+\partial_{k} \tilde{R}^{k}\left(p^{2}\right)\left[\frac{\delta G_{k}}{\delta \chi^{a}(p)} \frac{\delta G_{k}}{\delta \bar{\chi}^{a}(-p)}-\frac{\delta^{2} G_{k}}{\delta \chi^{a}(p) \delta \bar{\chi}^{a}(-p)}\right]\right\} .
\end{aligned}
$$

Next we perform a Legendre transformation in order to arrive at the effective action $\Gamma_{k}(A, c, \bar{c}, K, L)$. Here $A, c$ and $\bar{c}$ denote classical fields, and the sources $K$ and $L$ are the same as before:

$$
\begin{aligned}
G_{k}(J, \chi, \bar{\chi}, K, L) & =\Gamma_{k}(A, c, \bar{c}, K, L)-(J \cdot A+\bar{\chi} \cdot c+\chi \cdot \bar{c}), \\
A_{\mu}{ }^{a} & =-\frac{\delta G_{k}}{\delta J_{\mu}{ }^{a}}, \quad c^{a}=-\frac{\delta G_{k}}{\delta \bar{\chi}^{a}}, \quad \bar{c}^{a}=-\frac{\delta G_{k}}{\delta \chi^{a}}, \\
J_{\mu}{ }^{a} & =\frac{\delta \Gamma_{k}}{\delta A_{\mu}{ }^{a}}, \quad \bar{\chi}^{a}=-\frac{\delta \Gamma}{\delta c^{a}}, \quad \chi^{a}=-\frac{\delta \Gamma}{\delta \bar{c}^{a}} .
\end{aligned}
$$

After the Legendre transformation the second variations of $G_{k}$ with respect to sources, as present in eq. (14), can be expressed in terms of $\Gamma_{k}$. Essentially they are given by the inverse of the second variations of $\Gamma_{k}$ with respect to the fields; 
the inverse has to be formed, however, in the enlarged space spanned by the three fields $\{A, c, \bar{c}\}$. The precise relations, including the correct signs, can most easily be obtained by varying the second line of eq. (15) with respect to the three fields, and by expressing the variations of $G_{k}$ with respect to the fields in terms of variations with respect to the sources using the third line of eq. (15). It becomes convenient to define

$$
\frac{\delta^{2} \Gamma_{k}^{-1}}{\delta \varphi^{a} \delta \varphi^{b}} \equiv-\frac{\delta^{2} G_{k}}{\delta j^{a} \delta j^{b}}
$$

where $\varphi^{a}$ denotes $\left\{A_{\mu}{ }^{a}, c^{a}, \bar{c}^{a}\right\}$ in the cases where $j^{a}$ is given by $\left\{J_{\mu}{ }^{a}, \chi^{a}, \bar{\chi}^{a}\right\}$, respectively. (Note the association of $c^{a}$ with $\chi^{a}$ and not $\bar{\chi}^{a}$; below it will be useful to define $\bar{\varphi}^{a}=\left\{A_{\mu}^{a}, \bar{c}^{a}, c^{a}\right\}$ in terms of $\varphi^{a}=\left\{A_{\mu}^{a}, c^{a}, \bar{c}^{a}\right\}$.) Eq. (16) is to be read as a definition of the l.h.s. in terms of the r.h.s.; the l.h.s. is not to be identified with $\left(\delta^{2} \Gamma_{k} / \delta \varphi^{a} \delta \varphi^{b}\right)^{-1}$. Now the flow equations for $\Gamma_{k}(A, c, \bar{c}, K, L)$ can be expressed as

$$
\begin{aligned}
\partial_{k} \Gamma_{k}= & \int D p\left\{\frac{1}{2} \partial_{k} R_{\mu \nu}^{k}\left(p^{2}\right)\left[A_{\mu}^{a}(-p) A_{\nu}^{a}(p)+\frac{\delta^{2} \Gamma_{k}^{-1}}{\delta A_{\mu}^{a}(p) \delta A_{\nu}^{a}(-p)}\right]\right. \\
& \left.+\partial_{k} \tilde{R}^{k}\left(p^{2}\right)\left[\bar{c}^{a}(-p) c^{a}(p)-\frac{\delta^{2} \Gamma_{k}^{-1}}{\delta \bar{c}^{a}(p) \delta^{a} c(-p)}\right]\right\} .
\end{aligned}
$$

It assumes an even simpler form, if we introduce $\hat{\Gamma}_{k}$ by

$$
\Gamma_{k}=\hat{\Gamma}_{k}+\Delta S_{k}
$$

the flow equation for $\hat{\Gamma}_{k}$ simply reads

$$
\partial_{k} \hat{\Gamma}_{k}=\int D p\left\{\frac{1}{2} \partial_{k} R_{\mu \nu}^{k} \frac{\delta^{2} \Gamma_{k}^{-1}}{\delta A_{\mu}^{a}(p) \delta A_{\nu}^{a}(-p)}-\partial_{k} \tilde{R}^{k} \frac{\delta^{2} \Gamma_{k}^{-1}}{\delta \bar{c}^{a}(p) \delta c^{a}(-p)}\right\} .
$$

Next we turn to the modified ST identities. The starting point is again the path integral representation eq. (8) for $G_{k}$. We perform a field redefinition of the three fields $A \rightarrow A+\delta A, c \rightarrow c+\delta c, \bar{c} \rightarrow \bar{c}+\delta c$, where the variations have the form of BRS transformations (7). Since this field redefinition does not affect the complete expression, we obtain a condition for the vanishing of a sum of expectation values, which are not manifestly BRS invariant:

$$
\left\langle-\delta \Delta S_{k}+J \delta A+\bar{\chi} \delta c+\chi \delta \bar{c}\right\rangle=0 .
$$

After replacing fields by variations with respect to sources as before, and $\delta A, \delta c$ by variations with respect to $K, L$, we obtain the following identity for $G_{k}$ :

$$
\begin{aligned}
& \int D p\left\{J_{\mu}^{a}(p) \frac{\delta G_{k}}{\delta K_{\mu}^{a}(p)}+\bar{\chi}^{a}(p) \frac{\delta G_{k}}{\delta L^{a}(p)}+\frac{i}{\alpha} p_{\mu} \chi^{a}(p) \frac{\delta G_{k}}{\delta J_{\mu}{ }^{a}(p)}\right. \\
& +R_{\mu \nu}^{k}\left(p^{2}\right)\left[\frac{\delta G_{k}}{\delta K_{\nu}{ }^{a}(-p)} \frac{\delta G_{k}}{\delta J_{\mu}{ }^{a}(p)}-\frac{\delta^{2} G_{k}}{\delta K_{\nu}{ }^{a}(-p) \delta J_{\mu}{ }^{a}(p)}\right] \\
& +\tilde{R}^{k}\left(p^{2}\right)\left[\frac{\delta G_{k}}{\delta L^{a}(-p)} \frac{\delta G_{k}}{\delta \chi^{a}(p)}-\frac{\delta^{2} G_{k}}{\delta L^{a}(-p) \delta \chi^{a}(p)}\right] \\
& \left.+\frac{i}{\alpha} p_{\mu} \tilde{R}^{k}\left(p^{2}\right)\left[\frac{\delta G_{k}}{\delta J_{\mu}{ }^{a}(-p)} \frac{\delta G_{k}}{\delta \bar{\chi}^{a}(p)}-\frac{\delta^{2} G_{k}}{\delta J_{\mu}{ }^{a}(-p) \delta \bar{\chi}^{a}(p)}\right]\right\}=0 .
\end{aligned}
$$


The first line of eq. (21) corresponds to the standard ST identity; the additional terms involving $R_{\mu \nu}^{k}$ or $R^{k}$ are new and originate from the term $-\delta \Delta S_{k}$ in eq. (20).

Note that, because of the properties of the functions $R_{\mu \nu}^{k}$ and $\tilde{R}^{k}$ (cf. eqs. (11) and (13) $)$, the corresponding momentum integral $D p$ is bound to be ultraviolet-finite. Furthermore, for $k \rightarrow 0$, the functions $R_{\mu \nu}^{k}$ and $\tilde{R}^{k}$ vanish, and (21) turns into the standard ST identity. The equation involves just a one-loop momentum integral $D p$; this integration is free of infrared divergencies for non-exceptional (euclidean) external momenta. It will be helpful to assign a name to the functional of the sources appearing on the l.h.s. of eq. (21); we will denote the entire l.h.s. of eq. (21) by $\Sigma_{k}$.

Next we will have a look at the $k$-dependence of $\Sigma_{k}$. In evaluating $\partial_{k} \Sigma_{k}$, the $k$ derivative hits $R_{\mu \nu}^{k}, \tilde{R}^{k}$ and $G_{k}$ present in $\Sigma_{k}$; the $k$-derivative of $G_{k}$ is then replaced by the r.h.s. of the flow equation (14). After a straightforward, though lengthy calculation, the result can be written as

$$
\begin{aligned}
& \partial_{k} \Sigma_{k}=\int D p\left\{\partial_{k} R_{\mu \nu}^{k}\left(p^{2}\right)\left[\frac{\delta G_{k}}{\delta J_{\mu}{ }^{a}(-p)} \frac{\delta \Sigma_{k}}{\delta J_{\nu}{ }^{a}(p)}-\frac{1}{2} \frac{\delta^{2} \Sigma_{k}}{\delta J_{\mu}{ }^{a}(-p) \delta J_{\nu}{ }^{a}(p)}\right]\right. \\
& \left.+\partial_{k} \tilde{R}^{k}\left(p^{2}\right)\left[\frac{\delta G_{k}}{\delta \chi^{a}(-p)} \frac{\delta \Sigma_{k}}{\delta \bar{\chi}^{a}(p)}-\frac{\delta G_{k}}{\delta \bar{\chi}^{a}(-p)} \frac{\delta \Sigma_{k}}{\delta \chi^{a}(p)}-\frac{\delta^{2} \Sigma_{k}}{\delta \chi^{a}(-p) \delta \bar{\chi}^{a}(p)}\right]\right\} .
\end{aligned}
$$

Since the r.h.s. of (22) is linear in $\Sigma_{k}$, the following important statement holds: If $\Sigma_{k}$ vanishes for some $k=\bar{k}$ (identically in the sources), it will vanish for all $k$, provided $G_{k}$ and $G_{\bar{k}}$ are related by the integration of the flow equation (14). This allows us to forget about the derivation of eq. (21), and to start anew as follows:

Let us define $G_{k}$ by a functional satisfying the flow equation (14). Its path integral representation (8) is helpful for its physical interpretation, but not required for its definition. Let us now require that, at some starting point $k=\bar{k}, G_{\bar{k}}$ satisfies the modified ST identity (21), without referring to its derivation. Eq. (22) then ensures us that $G_{k}$ will satisfy eq. (21) for all $k$, in particular for $k \rightarrow 0$. Since eq. (21) turns into the standard ST identity for $k \rightarrow 0$, we thus obtain the desired property of $G_{k \rightarrow 0}$. The assumptions underlying the existence of the path integral representation (8) are not required any more.

Before we turn to the effective action, we observe that another useful identity can be derived in a similar fashion: If we perform a redefintion of the field $\bar{c}^{a}$ alone, and use that $\delta S_{\mathrm{gh}} / \delta \bar{c}^{a} \sim-\partial_{\mu} O_{A, \mu}^{a}$, we find a relation of the form

$$
i p_{\mu} \frac{\delta G_{k}}{\delta K_{\mu}^{a}(p)}-\chi^{a}(-p)+\tilde{R}^{k}\left(p^{2}\right) \frac{\delta G_{k}}{\delta \bar{\chi}^{a}(p)}=0
$$

Also this identity can be shown to be invariant under the RG flow. The form of the modified ST identity for $\Gamma_{k}$ can simply be obtained by inserting the Legendre transformations (15) into eq. (21), as in the case of the flow equations. We just need two more relations, in order to express the variations $\delta^{2} G_{k} / \delta K \delta J$ and $\delta^{2} G_{k} / \delta L \delta \chi$ in terms of $\Gamma_{k}$. These have the form

$$
\frac{\delta^{2} G_{k}}{\delta K_{\nu}{ }^{a}(-p) \delta J_{\mu}{ }^{a}(p)}=\int D q \sum_{b} \frac{\delta^{2} \Gamma_{k}^{-1}}{\delta A_{\mu}^{a}(p) \delta \varphi^{b}(q)} \frac{\delta^{2} \Gamma_{k}}{\delta \bar{\varphi}^{b}(-q) \delta K_{\nu}^{a}(-p)}
$$




$$
\equiv \sum_{\varphi^{b}} \frac{\delta^{2} \Gamma_{k}^{-1}}{\delta A_{\mu}^{a}(p) \delta \varphi^{b}} \frac{\delta^{2} \Gamma_{k}}{\delta \bar{\varphi}^{b} \delta K_{\nu}^{a}(-p)},
$$

and a similar equation with $K_{\nu}{ }^{a}$ replaced $L^{a} \cdot \delta^{2} \Gamma_{k}^{-1} / \delta A \delta \varphi^{b}$ and $\bar{\varphi}^{b}$ have been defined in and below eq. (16), respectively.

The ultimate form of the modified ST identity for the effective action becomes again simpler, if we express it in terms of $\hat{\Gamma}_{k}$ defined in eq. (18). It finally reads

$$
\begin{aligned}
& \int D p\left\{\frac{\delta \hat{\Gamma}_{k}}{\delta A_{\mu}^{a}(p)} \frac{\delta \hat{\Gamma}_{k}}{\delta K_{\mu}{ }^{a}(-p)}-\frac{\delta \hat{\Gamma}_{k}}{\delta c^{a}(p)} \frac{\delta \hat{\Gamma}_{k}}{\delta L^{a}(-p)}-\frac{i}{\alpha} p_{\mu} A_{\mu}^{a}(p) \frac{\delta \hat{\Gamma}_{k}}{\delta \bar{c}^{a}(p)}\right. \\
& -\sum_{\varphi^{b}}\left[R_{\mu \nu}^{k}\left(p^{2}\right) \frac{\delta^{2} \Gamma_{k}^{-1}}{\delta A_{\mu}^{a}(p) \delta \varphi^{b}} \frac{\delta^{2} \hat{\Gamma}_{k}}{\delta \bar{\varphi}^{b} \delta K_{\nu}{ }^{a}(-p)}+\tilde{R}^{k}\left(p^{2}\right) \frac{\delta^{2} \Gamma_{k}^{-1}}{\delta c^{a}(p) \delta \varphi^{b}} \frac{\delta^{2} \hat{\Gamma}_{k}}{\delta \bar{\varphi}^{b} \delta L^{a}(-p)}\right] \\
& \left.-\frac{i}{\alpha} p_{\mu} \tilde{R}^{k}\left(p^{2}\right) \frac{\delta^{2} \Gamma_{k}^{-1}}{\delta A_{\mu}^{a}(p) \delta \bar{c}^{a}(-p)}\right\}=0
\end{aligned}
$$

The relation (23) becomes in terms of $\hat{\Gamma}_{k}$

$$
i p_{\mu} \frac{\delta \hat{\Gamma}_{k}}{\delta K_{\mu}^{a}(p)}+\frac{\delta \hat{\Gamma}_{k}}{\delta \bar{c}^{a}(p)}=0
$$

and is thus not at all affected by the infrared cutoff $k$.

We will now present a consistency check of the formalism within lowest order in perturbation theory. As mentioned above we will concentrate on the mass term for the gauge fields within $\Gamma_{k}$.

Within lowest order perturbation theory the flow equation (19) is easily integrated with the result

$$
\begin{aligned}
\hat{\Gamma}_{k}=\hat{\Gamma}_{0} & +\frac{1}{2} \operatorname{Tr}\left[\ln \left(\frac{\delta^{2} \hat{\Gamma}_{0}}{\delta A_{\mu}^{a}(p) \delta A_{\nu}^{b}(q)}+R_{\mu \nu}^{k}\right)-\ln \left(\frac{\delta^{2} \hat{\Gamma}_{0}}{\delta A_{\mu}^{a}(p) \delta A_{\nu}^{b}(q)}\right)\right] \\
& -\operatorname{Tr}\left[\ln \left(\frac{\delta^{2} \hat{\Gamma}_{0}}{\delta \bar{c}^{a}(p) \delta c^{b}(q)}+\tilde{R}^{k}\right)-\ln \left(\frac{\delta^{2} \hat{\Gamma}_{0}}{\delta \bar{c}^{a}(p) \delta c^{b}(q)}\right)\right] .
\end{aligned}
$$

Here $\operatorname{Tr}$ denotes the trace over Lorentz and gauge group indices as well as integration over internal momenta with the measure $D p$ of eq. (9). Furthermore we made use of $R_{\mu \nu}^{k=0}=\tilde{R}^{k=0}=0$.

Let us now make an ansatz for $\hat{\Gamma}_{0}$ in the form of a classical Yang Mills action as given by eqs. (1)-(6), $\hat{\Gamma}_{0}=S_{Y M}+S_{g f}+S_{g h}-K \cdot O_{A}-L \cdot O_{g}$. After inserting this ansatz for $\hat{\Gamma}_{0}$ into the r.h.s. of eq. (27), an expansion of eq. (27) up to second order in the gauge fields $A_{\mu}^{a}(p)$ and to zeroth order in the corresponding momentum $p$, one finds that $\hat{\Gamma}_{k}$ has to contain a mass term for the gauge fields of the form $\frac{1}{2} m^{2} A_{\mu}^{a} A_{\mu}^{a}$. Three diagrams with two external gauge fields contribute to $m^{2}$ : A ghost loop, a gauge field loop with one quartic vertex and a gauge field loop with two cubic vertices. The result for $m^{2}$ reads in $d=4$ dimensions, and with the form (13) of the the cutoff function $R\left(p^{2}\right)$ :

$$
m^{2}=\frac{3 N g^{2}}{128 \pi^{2}} k^{2}(\alpha-1)
$$


where $\alpha$ denotes the gauge parameter.

Likewise, the modified ST identity (25) can be solved within a perturbative expansion. To one loop order, $\Gamma_{k}$ or $\hat{\Gamma}_{k}$ can be replaced by $\Gamma_{0}$ resp. $\hat{\Gamma}_{0}$ in all terms appearing with factors of $R_{\mu \nu}^{k}$ or $\tilde{R}^{k}$. After an expansion of eq. (25) to first order in the gauge field $A_{\mu}^{a}(p)$, to first order in the ghost field $c^{a}(-p)$ and to first order in the momentum $p_{\mu}$ only the first term $\delta \hat{\Gamma}_{k} / \delta A_{\mu}^{a}(p) \times \delta \hat{\Gamma}_{k} / \delta K_{\mu}^{a}(-p)$ out of the first three "classical" terms survives (note that $\hat{\Gamma}_{k}$ can be assumed to be local due to the presence of the infra-red cutoff). Furthermore $\hat{\Gamma}_{k}$ within the second factor of this term can be replaced by $\hat{\Gamma}_{0}$, and the first factor is proportional to the gauge field mass term mentioned above.

Together with the numerous terms with factors of $R_{\mu \nu}^{k}$ or $\tilde{R}^{k}$ the modified ST identity (25) thus provides us with a one loop equation for the gauge field mass term. In $d$ dimensions, and for an arbitrary cutoff function $R^{k}$ in eqs. (11), this equation becomes

$$
\begin{aligned}
m^{2}= & \frac{N g^{2}}{16 \pi^{2}} \int_{0}^{\infty} d p^{2}\left(p^{2}\right)^{\frac{d}{2}-2} \frac{R^{k}\left(p^{2}\right)}{\left(1+R^{k}\left(p^{2}\right)\right)^{2}}\left\{\frac{11}{2}-d-\frac{5}{d}+\alpha\left(1-\frac{1}{d}\right)\right. \\
& \left.+\frac{p^{2} \partial_{p^{2}} R_{k}\left(p^{2}\right)}{\left(1+R^{k}\left(p^{2}\right)\right)}\left(\frac{7}{2}-\frac{6}{d}\right)\right\} .
\end{aligned}
$$

Using $d=4$ and the form (13) of the cutoff function $R^{k}\left(p^{2}\right)$, one recovers the result (28) for $m^{2}$ derived via the integration of the flow equations. This constitutes a nontrivial check of our formalism; at the diagrammatic level the contributions to eqs. (28) and (29) are entirely different. (Using partial integrations under the $d p^{2}$ integral, the equivalence of the different contributions can already be established in arbitrary dimensions and for arbitrary cutoff functions. More details will follow in a separate publication.)

Of course, the procedure underlying this consistency check required the knowledge of a solution of the standard ST identities, eq. (25) for $k \rightarrow 0$, in the form of the classical action. In the case of applications, which are envisaged in the future, this knowledge is not available; $\hat{\Gamma}_{k}$ for $k \rightarrow 0$ is the object to be computed in terms of $\hat{\Gamma}_{k}$ for $k=\Lambda$ by integrating the flow equations.

To this end solutions to the modified ST identities (25) have to be found; these can at least be constructed perturbatively along the lines discussed in [5] for small coupling or large $\Lambda$ due to asymptotic freedom.

To conclude, the here presented modified ST identities provide an essential step to treat non-abelian gauge theories with the help of flow equations. These have already been proven to be a powerful and flexible method elsewhere, but their applicability to gauge theories has been considered with scepticism before.

The flow equations together with the modified ST identities might even be able to serve as a nonperturbative definition of gauge theories; therefore, however, the structure of the solutions for $k \rightarrow 0$ has to be studied in detail. This problem is presently under investigation.

It should be clear that additional matter can be included, using the same methods and arguments discussed explicitly above, as long as the theory remains asymptot- 
ically free. Finally, our results might also be of help concerning the program of proofing renormalizability of gauge theories within the framework of flow equations.

\section{References}

[1] K. G. Wilson and I. Kogut, Phys. Rep. 12 (1974) 75;

F. Wegner, in: Phase Transitions and Critical Phenomena, Vol. 6, eds. C. Domb and M. Green (Academic Press, New York 1975).

[2] J. Polchinski, Nucl. Phys. B231 (1984) 269.

[3] B. Warr, Ann. of Phys. 183 (1988) 1 and 59;

T. Hurd, Comm. Math. Phys. 124 (1989) 153;

G. Keller, C. Kopper, and M. Salmhofer, Helvet. Phys. Acta 65 (1992) 32;

M. Bonini, M. D'Attanasio, and G. Marchesini, Nucl. Phys. B409 (1993) 441;

G. Keller and C. Kopper, Comm. Math. Phys. 161 (1994) 515;

R. Ball and R. Thorne, Oxford preprint OUTP-93-23, CERN preprints TH.7233/94 and 7234/94.

[4] G. Keller and C. Kopper, Phys. Lett. B273 (1991) 323.

[5] C. Becchi, Lectures given at the Summer School in Theoretical Physics Parma 1991, eds. M. Bonini, G. Marchesini and E. Onofri.

[6] M. Bonini, M. D’Attanasio, and G. Marchesini, Nucl. Phys. B418 (1994) 81, Parma preprints UPRF 93-388 and 94-392.

[7] C. Wetterich, Z. Phys. C57 (1993) 451; Phys. Lett. B301 (1993) 90, Z. Phys. C 60 (1993) 461;

U. Ellwanger, Z. Phys. C58 (1993) 619 and Heidelberg preprint HD-THEP-9330 (to appear in Z. Phys. C);

U. Ellwanger and L. Vergara, Nucl. Phys. B398 (1993) 52;

N. Tetradis and C. Wetterich, Nucl. Phys. B398 (1993) 659 and Heidelberg preprints HD-THEP-93-28, HD-THEP-93-36;

T. Clark, B. Haeri, and S. T. Love, Nucl. Phys. B402 (1993) 628;

T. Morris, CERN preprints TH.6977/93 and TH.7203/94;

P. Haagensen, Y. Kubyshin, J. Latorre, and E. Moreno, Phys. Lett. B323 (1994) 330;

U. Ellwanger and C. Wetterich, Heidelberg preprint HD-THEP-94-01 (to appear in Nucl. Phys. B).

[8] M. Reuter and C. Wetterich, Nucl. Phys. B391 (1993) 147; Nucl. Phys. B408 (1993) 91; Nucl. Phys. B417 (1994) 181.

[9] G. Kleppe and R. Woodard, Nucl. Phys. B388 (1992) 81.

[10] A. Slavnov, Theor. and Math. Phys. 10 (1972) 99;

J. C. Taylor, Nucl. Phys. B33 (1971) 436. 
[11] C. Becchi, A. Rouet, and R. Stora, Comm. Math. Phys. 42 (1975) 127; Ann. Phys. 98 (1976) 287. 